\title{
Mössbauer study of Fe-Re alloys prepared by mechanical alloying
}

\author{
R. Konieczny ${ }^{1} \cdot$ R. Idczak ${ }^{1}$
}

Published online: 18 February 2016

(C) The Author(s) 2016. This article is published with open access at Springerlink.com

\begin{abstract}
The room temperature Mössbauer spectra of ${ }^{57} \mathrm{Fe}$ were measured for nanocrystalline iron-based solid solutions $\mathrm{Fe}_{1-x} \mathrm{Re}_{x}$, prepared by mechanical alloying with $x$ in the range $0.01 \leq x \leq 0.04$. The obtained data were analysed in terms of the binding energy $E_{\mathrm{b}}$ between two rhenium atoms in the Fe-Re system. The extrapolated value of $E_{\mathrm{b}}$ for $x=0$ was used for computation of enthalpy of solution of rhenium in iron. The result was compared with that resulting from the cellular atomic model of alloys by Miedema as well as with value, derived from proper data for Fe-Re solid solutions obtained by melting in an arc furnace. From the comparison it follows that our findings are in agreement with the Miedema's model predictions and previous Mössbauer studies.
\end{abstract}

Keywords Mechanical alloying · Mössbauer spectroscopy · Binding energy $\cdot$ Enthalpy of solution · Nanocrystalline materials

\section{Introduction}

Nanocrystalline materials characterized by crystallite sizes of a few nanometres and a high grain boundary density have been intensively investigated in the last twenty years [1-4]. The impressive progress in the ball milling technique enables to produce a great variety such materials as nanocrystalline solid solutions, amorphous alloys or composites with unique properties. Moreover, some properties of nanocrystals are remarkably different than those of the bulk material. On the other hand the controlled thermal treatment of mechanically alloyed products combined with a structural investigation allow determining the structural

This article is part of the Topical Collection on Proceedings of the International Conference on the Applications of the Mössbauer Effect (ICAME 2015), Hamburg, Germany, 13-18 September 2015.

R. Konieczny

robi@ifd.uni.wroc.pl

1 Institute of Experimental Physics, University of Wroclaw, pl. M. Borna 9, 50-204 Wrocław, Poland 
stability of the materials. Monitoring of mechanical alloying can be performed by many different techniques, in particular by Mössbauer spectroscopy delivering information on, for example, the processes of the formation of the dilute solid solutions as well as structural changes, phase transformations, magnetic and thermodynamic properties of the materials [5-11]. In general, the Mössbauer spectroscopy is especially powerful when the appearance of impurity atoms in the vicinity of the Mössbauer probe have a sufficiently large effect on the hyperfine field generated at the probe, to yield distinguishable components in the Mössbauer spectrum attributed to different configurations of the probe neighbours. From the data given in the literature (see [12] for example) it follows that there are many binary iron systems suitable for those studies. The fact is worth noticing as the impurity interactions are simply related to the enthalpy of solution of the impurity elements in iron matrix [13] and the enthalpy concerning usually about $700 \mathrm{~K}$, is widely used in developing and testing different models of binary alloys as well as methods for calculating the alloy parameters [14-17]. Moreover, the Mössbauer spectroscopy findings concerning the enthalpy in some cases can be useful to verify the corresponding experimental data derived from the calorimetric studies $[18,19]$.

In this study, we used the ${ }^{57} \mathrm{Fe}$ Mössbauer spectroscopy for determination of hyperfine interactions of the ${ }^{57} \mathrm{Fe}$ nuclei in and some thermodynamic properties of nanocrystalline Fe-Re alloys prepared by mechanosynthesis and then thermally annealed. Rhenium impurities were selected due to the fact that substitution of Fe atoms from the two first coordination shells of ${ }^{57} \mathrm{Fe}$ Mössbauer probe by Re ones, essentially influences the hyperfine field $B$ at the probe (see [12] for example), which facilitates the analysis of the measured spectra. Thanks to that the obtained experimental data on the enthalpy of solution of Re in ferromagnetic Fe at about $700 \mathrm{~K}$, could be credible enough for comparison to future corresponding theoretical values as in the case of the Fe-Mo system [17]. According to our knowledge there are no theoretical calculations and calorimetric data concerning the enthalpy.

\section{Experimental and results}

\subsection{Samples preparation and measurements}

Powders of $99.98 \%$ pure iron and $99.99 \%$ pure rhenium with particle sizes less than $0.1 \mathrm{~mm}$ were mixed in order to get several $\mathrm{Fe}_{1-x} \mathrm{Re}_{x}$ systems with $x$ in the range $0.01 \leq x \leq 0.04$. The mechanical alloying processes were performed in a Fritsch Pulverisette 6 planetary ball mill with stainless-steel vial and balls. The total weight of the powders was $10 \mathrm{~g}$ and the ball-to-powder weight ratio was 10:1. The milling processes were carried out at room temperature under an argon atmosphere and the milling speed amounted to 500 revolutions per minute.

The room temperature ${ }^{57} \mathrm{Fe}$ Mössbauer spectra for the studied samples were measured by means of a constant-acceleration POLON spectrometer of standard design. The measurements for each specimen were performed twice, just after its synthesis in the mill and then after an annealing process. All samples were annealed in vacuum at $1270 \mathrm{~K}$ for $2 \mathrm{~h}$. After that they were slowly cooled to room temperature during $6 \mathrm{~h}$. Under these conditions, diffusion effectively stops at a certain temperature being about $700 \mathrm{~K}$ [10], so the observed distributions of atoms in the annealed specimens should be the frozen-in state corresponding to the temperature. The Mössbauer spectra for the samples measured after the annealing process are presented in Fig. 1. 


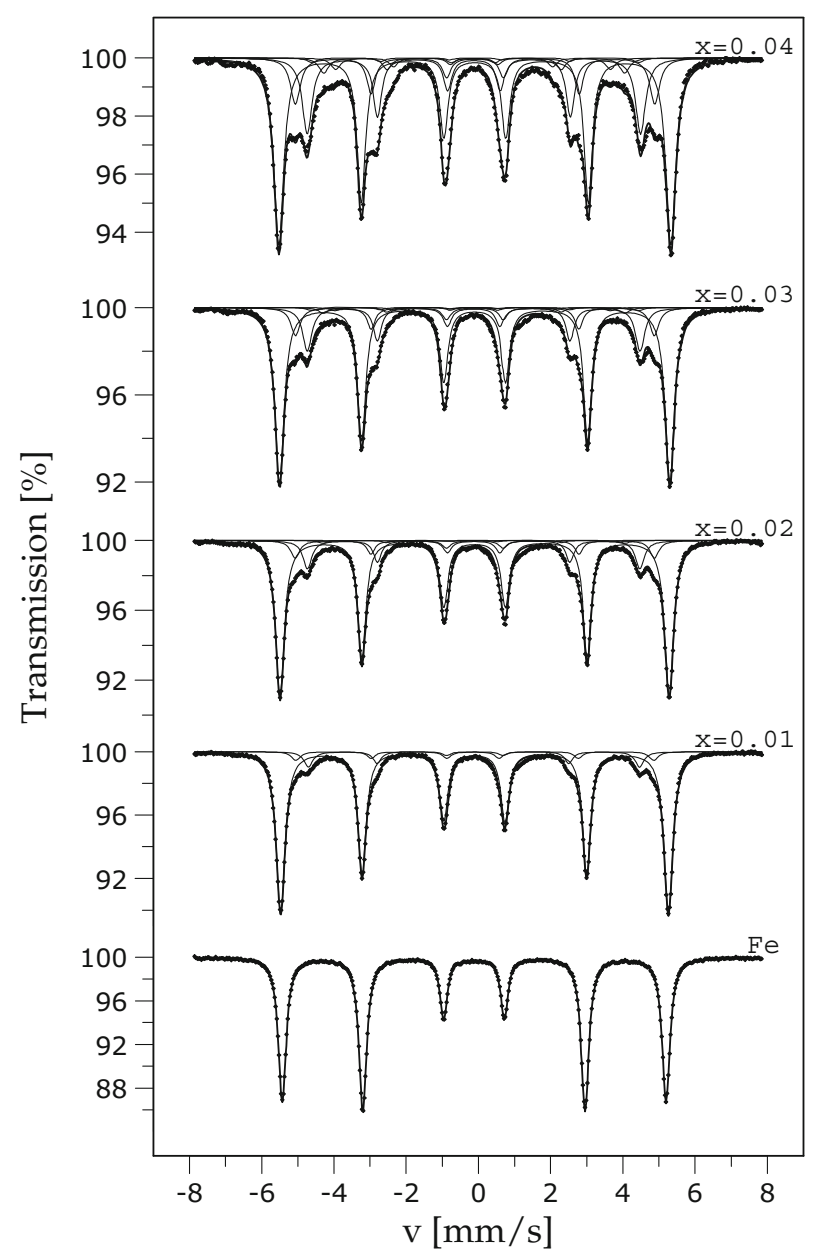

Fig. 1 The ${ }^{57} \mathrm{Fe}$ Mössbauer spectra for the nanocrystalline $\mathrm{Fe}_{1-x} \mathrm{Re}_{x}$ alloys measured at room temperature after the annealing process at $1270 \mathrm{~K}$

\subsection{Data analysis}

All measured Mössbauer spectra were fitted with a sum of certain number of different sixline patterns corresponding to unlike hyperfine fields $B$ at ${ }^{57} \mathrm{Fe}$ nuclei generated by different numbers of $\mathrm{Fe}$ and $\mathrm{Re}$ atoms located in the first two coordination shells of the probing nuclei. The number of fitted patterns increased with concentration of $\mathrm{Re}$ in the powder samples. The fitting procedure was done under assumption that the influence of rhenium atoms on $B$ as well as the corresponding isomer shift $I S$ of a subspectrum, is additive [20, 21] and independent of the atom positions in the given coordination shell of the nuclear probe although it can be different for atoms located in various shells. In other words it was accepted that for each subspectrum the quantities $B$ and $I S$ are linear functions of the 


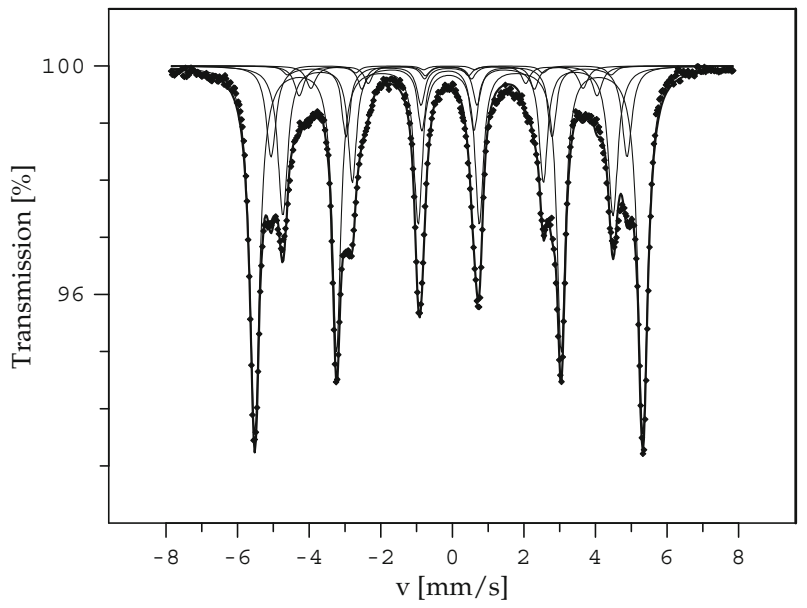

Fig. 2 The ${ }^{57} \mathrm{Fe}$ Mössbauer spectrum for the $\mathrm{Fe}_{0.96} \mathrm{Re}_{0.04}$ alloy measured at room temperature after the annealing process at $1270 \mathrm{~K}$, fitted with six six-line subspectra

numbers $n_{1}$ and $n_{2}$ of Re atoms located, respectively, in the first and second coordination shells of ${ }^{57} \mathrm{Fe}$ and the functions can be written as follows:

$$
\begin{aligned}
B\left(n_{1}, n_{2}\right) & =B_{0}+n_{1} \Delta B_{1}+n_{2} \Delta B_{2}, \\
I S\left(n_{1}, n_{2}\right) & =I S_{0}+n_{1} \Delta I S_{1}+n_{2} \Delta I S_{2},
\end{aligned}
$$

where $\Delta B_{1}\left(\Delta I S_{1}\right)$ and $\Delta B_{2}\left(\Delta I S_{2}\right)$ stand for the changes of $B(I S)$ with one Re atom in the first and second coordination shell of the Mössbauer probe. At the same time we assume that the quadruple shift $Q S$ in a cubic lattice is equal to zero because of the cubic symmetry of the investigated iron-based alloys [22] and the three linewidths $\Gamma_{16}, \Gamma_{25}$ and $\Gamma_{34}$ for all components of a spectrum which are related to the existence of impurity atoms in the two first coordination shells of ${ }^{57} \mathrm{Fe}$ are the same and they are different from linewidths of that component, determined by the nuclear probes having in their vicinity only Fe atoms. This division of subspectra into two sets with different linewidths takes into account the fact observed not only for this system, that the linewiths can be influenced by an existence of large/small enough impurity atoms in the nearest neighbourhood of the Mössbauer probes. The example of a fit obtained for the $\mathrm{Fe}_{0.96} \operatorname{Re}_{0.04}$ spectrum under assumptions mentioned above is presented in Fig. 2 whereas the values of the best-fit parameters for annealed systems are listed in Table 1 . The values are similar to corresponding data given in the literature [11, 23, 24]. For example in Ref. [11] one can find that for the $\mathrm{Fe}_{0.98} \mathrm{Re}_{0.02}$ alloy $\Delta B_{1}=-4.72(3) \mathrm{T}, \Delta B_{2}=-2.25(5) \mathrm{T}$ and $\Delta I S_{1}=-0.029(3) \mathrm{mm} / \mathrm{s}$, $\Delta I S_{2}=-0.003(7) \mathrm{mm} / \mathrm{s}$. In our previous work [23] concerning Fe-Re solid solutions obtained by melting we found that the nearest neighbour single Re impurity reduces hyperfine magnetic field on the iron nucleus by $-4.752 \mathrm{~T}$, while the next nearest neighbour single Re impurity decreases this field by $-2.502 \mathrm{~T}$. At the same time theoretical calculations based on density functional theory (DFT) give corresponding parameters which absolute values are lower than the experimental ones; according to the calculations $\Delta B_{1}=-3.27 \mathrm{~T}$, $\Delta B_{2}=-1.71 \mathrm{~T}, \Delta I S_{1}=-0.028 \mathrm{~mm} / \mathrm{s}$ and $\Delta I S_{2}=0.012 \mathrm{~mm} / \mathrm{s}$. This is due to the fact that DFT calculations for magnetic hyperfine field and isomer shift on the iron nucleus were performed for the isolated impurity located at various coordination shells around the 
Table 1 Some of the best-fit parameters of the assumed model of the ${ }^{57} \mathrm{Fe}$ Mössbauer spectrum measured for $\mathrm{Fe}_{1-x} \mathrm{Re}_{x}$ alloys after the annealing process. The standard uncertainties for the parameters result from the variance of the fit

\begin{tabular}{llllll}
\hline$x$ & $B_{0}[\mathrm{~T}]$ & $\Delta B_{1}[\mathrm{~T}]$ & $\Delta B_{2}[\mathrm{~T}]$ & $\Delta I S_{1}[\mathrm{~mm} / \mathrm{s}]$ & $\Delta I S_{2}[\mathrm{~mm} / \mathrm{s}]$ \\
\hline 0.01 & $33.2653(32)$ & $-4.864(36)$ & $-2.581(68)$ & $-0.0272(43)$ & $0.0095(10)$ \\
0.02 & $33.3795(41)$ & $-4.862(25)$ & $-2.570(41)$ & $-0.0251(28)$ & $0.0070(12)$ \\
0.03 & $33.4757(47)$ & $-4.931(21)$ & $-2.704(33)$ & $-0.0277(24)$ & $0.0067(9)$ \\
0.04 & $33.6220(64)$ & $-5.025(18)$ & $-2.796(28)$ & $-0.0254(19)$ & $0.0066(15)$ \\
\hline
\end{tabular}

iron atom in the bcc lattice filled with iron atoms otherwise. Moreover, calculations does not consider spin-orbit coupling or orbital contributions to the hyperfine field [25].

As the main result of the above analysis the values of parameters $c_{1}$ and $c_{2}$ of each spectrum were determined. Assuming that the Lamb-Mössbauer factor is independent of the configuration of atoms in the surroundings of the ${ }^{57} \mathrm{Fe}$ nucleus, the parameters are total intensities of those components of a spectrum which are related to the existence of one and two Re atoms in the two first coordination shells of nuclear probes ${ }^{57} \mathrm{Fe}$, respectively.

$$
\begin{aligned}
& c_{1}=c(1,0)+c(0,1), \\
& c_{2}=c(2,0)+c(1,1)+c(0,2) .
\end{aligned}
$$

The results are presented in Fig. 3 and listed in Table 2 together with calculated probabilities of finding zero $\mathrm{p}(0)$, one $\mathrm{p}(1)$ and two $\mathrm{p}(2)$ Re atoms in the two first coordination shells of an $\mathrm{Fe}$ atom in the random bcc $\mathrm{Fe}_{1-x} \mathrm{Re}_{x}$ alloy.

\subsection{The binding energy of two rhenium atoms in iron matrix}

Basing on the intensities of the spectrum components $c_{1}$ and $c_{2}$ we determined the binding energy $E_{\mathrm{b}}$ for pairs of rhenium atoms in the studied mechanically synthesized materials (Table 2). The computations were performed on the basis of the modified HrynkiewiczKrólas formula [26, 27] assuming that the ratio of concentrations of the individual pairs of $\mathrm{Re}$ atoms and isolated $\mathrm{Re}$ atoms in the $\mathrm{Fe}-\mathrm{Re}$ system is equal to the quotient of intensities $c_{2}$ and $c_{1}$.

$$
E_{\mathrm{b}}=-k T_{\mathrm{d}} \cdot \ln \left[\left(1+2 \cdot c_{2} / c_{1}\right) \cdot\left(c_{2} / c_{1}\right) \cdot(1+2 \cdot p(2) / p(1))^{-1} \cdot(p(2) / p(1))^{-1}\right],
$$

where $k$ is the Boltzmann constant, $T_{\mathrm{d}}$ denotes the 'freezing' temperature for the atomic distribution in the sample $\left(T_{\mathrm{d}}=700 \mathrm{~K}\right), p(n)=[N ! /((N-n) ! n !)] x^{n}(1-x)^{N-n}$ is the probability for the existence of $n \mathrm{Re}$ atoms among all $N=14$ atoms located in the two first coordination shells $\left(n=1\right.$ or 2 ) of the Fe atom in a random $\mathrm{Fe}_{1-x} \mathrm{Re}_{x}$ alloy and $x$ stands for the concentration of Re atoms.

\subsection{An enthalpy of solution of rhenium in iron}

In the next step we found the extrapolated value of $E_{\mathrm{b}}$ for $x=0$ using $E_{\mathrm{b}}(0.02)$ and $E_{\mathrm{b}}(0.03)$. Such procedure was determined by the fact that the applied Hrynkiewicz-Królas method of the $E_{\mathrm{b}}$ estimation was developed for very dilute alloy, with $x$ close to zero. 


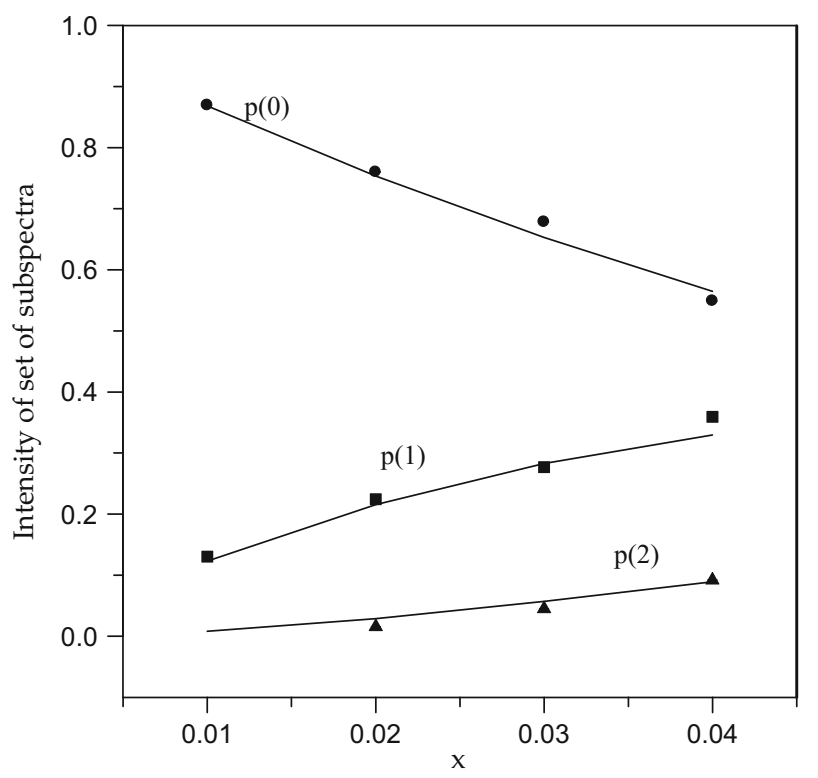

Fig. 3 Total intensities of those components of a spectrum which are related to the existence of zero $\left(c_{0}\right.$ - circular points), one ( $c_{1}$ - square points) and two $\left(c_{2}\right.$ - triangular points) Re atoms in the two first coordination shells of nuclear probes ${ }^{57} \mathrm{Fe}$ in the $\mathrm{Fe}_{1-x} \mathrm{Re}_{x}$ alloys after the annealing process. The solid lines describe probabilities of finding zero - $p(0)$, one - $p(1)$ and two - $p(2)$ atoms $\mathrm{Re}$ in the two first coordination shells $(N=14)$ of an $\mathrm{Fe}$ atom in the binomial bcc $\mathrm{Fe}_{1-x} \mathrm{Re}_{x}$ alloy

Table 2 The binding energy $E_{\mathrm{b}}$ between a pair of Re atoms in mechanosynthesized $\mathrm{Fe}_{1-x} \mathrm{Re}_{x}$ alloys after the annealing process deduced from the ${ }^{57} \mathrm{Fe}$ Mössbauer spectra. The standard uncertainties for $c_{1}$ and $c_{2}$ result from the variance of the fit of the assumed model to the spectrum measured. The values of uncertainty for $E_{\mathrm{b}}$ were computed assuming that the uncertainty for the 'freezing' temperature $T_{\mathrm{d}}$ is $50 \mathrm{~K}$

\begin{tabular}{llllll}
\hline$x$ & $c_{1}$ & $c_{2}$ & $p(1)$ & $p(2)$ & $E_{\mathrm{b}}(\mathrm{eV})$ \\
\hline 0.01 & $0.1303(34)$ & - & 0.1229 & 0.0081 & - \\
0.02 & $0.2244(39)$ & $0.0154(34)$ & 0.2153 & 0.0286 & $0.0461(36)$ \\
0.03 & $0.2769(40)$ & $0.0449(44)$ & 0.2827 & 0.0568 & $0.0165(14)$ \\
0.04 & $0.3592(44)$ & $0.0920(45)$ & 0.3294 & 0.0892 & $0.0045(5)$ \\
\hline
\end{tabular}

Finally, the $E_{\mathrm{b}}(0)$ equal to $0.106(11) \mathrm{eV}$ was used for computation of the enthalpy of solution $H_{\mathrm{Fe}-\mathrm{Re}}$ of $\mathrm{Re}$ in iron. The calculations were performed on the basis of the Królas model [13] for the binding energy according to which

$$
H_{\mathrm{Fe}-\mathrm{Re}}=-\mathrm{z} \cdot E_{\mathrm{b}}(0) / 2
$$

where $\mathrm{z}$ is the coordination number of the crystalline lattice ( $\mathrm{z}=8$ for $\alpha-\mathrm{Fe}$ ). The obtained value for $H_{\mathrm{Fe}-\mathrm{Re}}$, equal to -0.424(44) eV/atom, was compared with the corresponding value $-0.015 \mathrm{eV} /$ atom, resulting from the semiempirical Miedema's model of alloys [14] as well as with that -0.22 (13) eV/atom [23], derived from proper Mössbauer data for Fe-Re solid solutions obtained by melting in an arc furnace. The result is displayed in Table 3. 
Table 3 The enthalpy $H_{\mathrm{Fe}-\mathrm{Re}}$ [eV/atom] of solution of rhenium in iron. The value of uncertainty for enthalpy $H_{\mathrm{Fe}-\mathrm{Re}}$ was computed as the composed standard uncertainty; in the case of mechanical alloying the uncertainty for the extrapolated value of binding energy $E_{\mathrm{b}}(0)$ is $0.011 \mathrm{eV}$, while for melting alloys it is $0.033 \mathrm{eV}$

\begin{tabular}{llc}
\hline Miedema's model [14] & Melting alloys [23] & Mechanical alloying \\
\hline-0.015 & $-0.22(13)$ & $-0.424(44)$ \\
\hline
\end{tabular}

\section{Conclusions}

The positive value of the binding energy $E_{\mathrm{b}}$ between two rhenium atoms in the studied mechanosynthesized Fe-Re alloys after the thermal annealing, speaks in favour of the suggestion that $\mathrm{Re}$ atoms interact repulsively in nanocrystalline iron matrix. The value $-0.424(44) \mathrm{eV} /$ atom for the enthalpy of solution $H_{\mathrm{Fe}-\mathrm{Re}}$ of rhenium in $\alpha$-iron, determined from the ${ }^{57} \mathrm{Fe}$ Mössbauer spectra are in a qualitative agreement with that $-0.015 \mathrm{eV} /$ atom, resulting from the Miedema's model of alloys which is based on hundreds calorimetric data concerning the heat of formation for different binary systems as well as this derived from our previous data for $\mathrm{Fe}-\mathrm{Re}$ solid solutions obtained by melting in an arc furnace. However taking into account the uncertainties of the determined $H_{\mathrm{Fe}-\mathrm{Re}}$ values one can say that the enthalpy of solution is practically the same for two different ways of forming the solution melting and mechanical synthesis.

The obtained results support the suggestion that the applied ${ }^{57} \mathrm{Fe}$ Mössbauer spectroscopy method for investigation of thermodynamic properties of alloys is a good tool in the case of mechanically synthesized binary, iron-based solid solutions, and it can supply experimental data useful, in particular, for verification of theoretical works.

Acknowledgments This work was supported by the University of Wrocław under the grant 1429/M/IFD/15

Open Access This article is distributed under the terms of the Creative Commons Attribution 4.0 International License (http://creativecommons.org/licenses/by/4.0/), which permits unrestricted use, distribution, and reproduction in any medium, provided you give appropriate credit to the original author(s) and the source, provide a link to the Creative Commons license, and indicate if changes were made.

\section{References}

1. Takeuchi, J., Katsube, H., Hiromitsu, I., Ito, T.: Magnetic properties of mechanically alloyed amorphous Fe-M-Zr (M = Mn, Co, Ni, Ce, Dy, Er). J. Magn. Magn. Mater. 140-144, 303-304 (1995)

2. Froesa, F.H., Suryanarayana, C., Russellb, K., Li, C.-G.: Synthesis of intermetallics by mechanical alloying. Mater. Sci. Eng. A 192(/193), 612-623 (1995)

3. Suryanarayana, C.: Mechanical alloying and milling. Prog. Mater. Sci. 46, 1-184 (2001)

4. Shen, T.D., Xin, S.W., Sun, B.R.: Influence of annealing on the mechanical property of iron- and nickelbased nanocrystalline alloys. Mater. Sci. Eng. A 627, 139-144 (2015)

5. Karolus, M., Jartych, E., Oleszak, D.: Structure and magnetic properties of nanocrystalline Fe-Mo alloys prepared by mechanosynthesis. Acta Phys. Pol. A 102, 253-258 (2001)

6. Konieczny, R., Idczak, R.: Atomic short-range order in mechanically synthesized iron based Fe-Zn alloys studied by ${ }^{57} \mathrm{Fe}$ Mössbauer spectroscopy. Nukleonika 60, 69-73 (2015)

7. Idczak, R., Konieczny, R., Chojcan, J.: Short-range order in iron alloys studied by ${ }^{57}$ Fe Mössbauer spectroscopy. Solid State Commun. 159, 22-25 (2013)

8. Konieczny, R., Idczak, R., Chojcan, J.: Mössbauer studies of interactions between osmium atoms dissolved in iron. Nukleonika 60, 75-79 (2015) 
9. Konieczny, R., Idczak, R., Elsner, J., Chojcan, J.: An enthalpy of solution of platinum in iron studied by ${ }^{57} \mathrm{Fe}$ Mössbauer spectroscopy. Hyperfine Interact. 206, 119 (2012)

10. Idczak, R., Konieczny, R., Chojcan, J.: An enthalpy of solution of chromium in iron studied with ${ }^{57} \mathrm{Fe}$ Mössbauer spectroscopy. Physica B 407, 2078-2081 (2012)

11. Idczak, R., Konieczny, R., Konieczna, Ż., Chojcan, J.: An Enthalpy of Solution of Cobalt and Nickel in Iron Studied with ${ }^{57}$ Fe Mössbauer Spectroscopy. Acta Phys. Pol. A 119, 37-40 (2011)

12. Vincze, I., Campbell, I.A.: Mössbauer measurements in iron based alloys with transition metals. J. Phys. F 3, 647 (1973)

13. Królas, K.: Correlation between impurity binding energies and heat of formation of alloys. Phys. Lett. A 85, 107 (1981)

14. Miedema, A.R.: Energy effects and charge transfer in metal physics, modeling in real space. Physica B 182, 1 (1992)

15. Bonny, G., Pasianot, R.C., Malerba, L., Caro, A., Olsson, P., Lavrentiev, M.Y.: Numerical predictions of thermodynamic properties of iron-chromium alloys using semi-empirical cohesive models: the state of the art. J. Nucl. Mater. 385, 268 (2009)

16. Boom, R., De Boer, F.R., Niessen, A.K., Miedema, A.R.: Enthalpies of formation of liquid and solid binary alloys based on 3d metals. Physica 115B, 285 (1983)

17. Lejaeghere, K., Cottenier, S., Claessens, S., Waroquier, M., Van Speybroeck, V.: Assessment of a lowcost protocol for an ab inito based prediction of the mixing enthalpy at elevated temperatures: the Fe-Mo system. Phys. Rev. B 83, 184201 (2011)

18. Hultgren, R., Desai, P.D., Hawkins, D.T., Gleiser, M., Kelley, K.K.: Selected Values of Thermodynamic Properties of Binary Alloys. American Society for Metals, Metals Park, Ohio (1973)

19. Swartzendruber, L.J., Itkin, V.P., Alcock, C.B.: Phase diagrams of binary iron alloys. In: Okamoto, H. (ed.) Materials Information Society. Materials Park, Ohio (1993)

20. Wertheim, G.K., Jaccarino, V., Wernick, J.H., Buchanan, D.N.E.: Range of the exchange interaction in iron alloys. Phys. Rev. Lett. 12, 24-27 (1964)

21. Sauer, W.E., Reynik, R.J.: Electronic and magnetic structure of dilute Iron-Base alloys. J. Appl. Phys. 42, 1604-1605 (1971)

22. Ovchinnikov, V.V.: Mössbauer Analysis of the Atomic and Magnetic Structure of Alloys. Cambridge International Science Publishing, Cambridge (2006)

23. Konieczny, R., Idczak, R., Szarypo, W., Chojcan, J.: An enthalpy of solution of rhenium in iron studied by ${ }^{57} \mathrm{Fe}$ Mössbauer spectroscopy. Hyperfine Interact. 206, 135 (2012)

24. Dubiel, S.M.: Rhenium-induced changes in the electronic structure of iron. J. Magn. Magn. Mater. 69, 206-214 (1987)

25. Błachowski, A., Wdowik, U.D.: Transition metal impurity effect on charge and spin density in iron: Ab initio calculations and comparison with Mössbauer data. J. Phys. Chem. Solids 73, 317-323 (2012)

26. Chojcan, J.: Interaction between impurity atoms of $3 \mathrm{~d}$ transition metals dissolved in iron. J. Alloys Compd. 264, 50 (1998)

27. Hrynkiewicz, A.Z., Królas, K.: Formation of two-impurity complex in dilute alloys observed through perturbed angular correlation of $\gamma$ rays. Phys. Rev. B 28, 1864-1869 (1983) 